\title{
Detection of glyphosate resistance in black nightshade Solanum nigrum from Hunan China
}

\author{
Tingting Zou, Chenzhong Jin, Zanjiang Zhu, Yihong $\mathbf{H u}^{*}$ \\ Collaborative Innovation Center for Field Weeds Control of Hunan Province, \\ Hunan University of Humanities, Science and Technology, Loudi 417000 China \\ *Corresponding author, e-mail: huyhongwangyi@163.com
}

Received 15 Oct 2018

Accepted 27 Sep 2019

\begin{abstract}
Black nightshade (Solanum nigrum) is a kind of common malignant weed in the agricultural fields worldwide. In recent years, it has not been well controlled under the commercial rate of glyphosate application in some areas of Hunan Province in China. To evaluate and confirm the glyphosate resistant biotypes of $S$. nigrum, the seeds of ten suspected glyphosate resistant populations of $S$. nigrum were collected in the agricultural fields from this province. Their GR50 values and glyphosate resistance were determined by the biomass method, the comprehensive index of pesticide injury method, and the leaf discs method independently. The results showed that ' $r$, r2, r3, and r9' populations were of moderate resistance levels to glyphosate. But the shikimic acid levels in S. nigrum did not display a typical sigmoidal curve to glyphosate resistance, supporting that the mechanism of different resistance might be involved among the glyphosate resistant biotypes in $S$. nigrum.
\end{abstract}

KEYWORDS: Solanum nigrum, glyphosate resistant populations, GR50, shikimic acid, Hunan China

\section{INTRODUCTION}

Glyphosate, one of the most important non-selective herbicide, is widely used in the agricultural field globally. In the past decades, the heavy use of glyphosate has resulted in the widespread glyphosate resistant weed occurrence and evolution worldwide $^{1}$. Since glyphosate was first put into use in 1970s, at least more than 30 species of weed biotypes have been confirmed for glyphosate resistance $^{2}$. Among them, only seven species were identified as the mechanism of resistance to 5-enolpyruvylshikimate-3-phosphate (EPSP) synthase inhibitor ${ }^{3}$, whereas the other mechanisms were discounted as possible such as interfering in metabolism, sequestration, or translocation ${ }^{4,5}$. As a kind of prominent herbicide with little soil activity as glyphosate is, this herbicide was introduced into China in mid-1980s. Since then it has been customarily applied before planting in China. Nowadays this heavy application of glyphosate has occurred with widespread glyphosate resistant weed populations in China and has begun to threaten the crops security.

Black nightshade (Solanum nigrum) is a kind of annual or perennial broadleaf weed belonging to Solanum genus of the Solanaceae family, which is widely distributed all over the world ${ }^{6}$. Apart from being used as a traditional herb for heart, inflammation, and cancer medications ${ }^{7,8}$, S. nigrum is also considered as an important malignant weed in many crop fields in agriculture, as commonly seen in fields, wastelands, roadsides, fruit farms, and open forests, owning to its characteristics with wide distribution, numerous seeds, strong seed shattering, and strong stress resistance to environment ${ }^{9,10}$. It may reduce both the quality and the quantity of crops by competition and contaminating the harvested products. For example, in the years of 2016 and 2017, a weed population survey by our group in the no-tillage juvenile oil tea (Camellia oleifera Abei) fields treated with tank mixtures of glyphosate and quizalofop-p-ethyl in Hunan Province of China revealed that some of the $S$. nigrum biotypes exhibited resistances to glyphosate and even survived and reproduced up to an additional lethal dosage of $1800 \mathrm{~g}$ ai/ha of glyphosate. This phenomenon is unacceptable both economically and environmentally in horticulture.

Among all reported glyphosate resistant weeds, most of them are belonging to the 'narrow leaf' species from the Gramineae family. At the same time, the broadleaf species are only found from the Comositae and Amaranthaceae families such as Amaranthus palmeri, Biden pilosa, and Conyza canadensis, etc ${ }^{11-13}$. S. nigrum is a weed species of 
great importance in agriculture, and our group has been studying on the $S$. nigrum populations in recent years. It was observed that some biotypes of them were not well controlled with the normal lethal dose of glyphosate in the agricultural fields. Up to now, no glyphosate resistant weeds from the Solanaceae family have been reported and registered ${ }^{2}$. Thus this study was developed to assess the resistance to glyphosate of $S$. nigrum from the Chinese biotypes.

\section{MATERIALS AND METHODS}

\section{Materials and chemicals}

The suspected glyphosate resistant seeds of ten S. nigrum populations collected in 2014 from the agricultural fields at Changde and Loudi areas of Hunan Province, China, were labelled from ' $r 1$ ' to 'r10', respectively. The susceptible seeds labelled 's' were stored in our laboratory. Glyphosate isopropyl amine was provided by Loudi Agriculture Science Institute, Hunan, China. The shikimic acid of high grade was purchased from Shanghai Baomanbio Co., and the other chemicals were of analytical grade.

\section{Seed germination and plant cultivation}

S. nigrum seeds were put onto the double-layer filter paper in Petri dishes, then were soaked with distilled water and bred at $25^{\circ} \mathrm{C}$ for $24 \mathrm{~h}$ first. After that moderate amount of $1.3 \% \mathrm{NaOH}$ was added to the seeds and bred at $25^{\circ} \mathrm{C}$ for $48 \mathrm{~h}$ to break the dormancy of $S$. nigrum seeds. After that, the seeds were washed with distilled water and transferred to an automatic environmental chamber (Model Conviron A-1000, Conviron Co., Winnipeg, Canada) to breed at $25^{\circ} \mathrm{C}$ with $65 \%$ relative humidity on a photoperiod of 14:10 light/dark cycle. After emergence, the seedlings were transplanted to the pots at one plant per pot with the commercial nutritive soil and bred on the same condition. Then the pots were transferred to breed outdoors at 4-6 leaf stage. A total of 41 pots per ' $r 1-\mathrm{r} 10$ ' population and 41 pots per 's' population were bred.

\section{Treatment with glyphosate}

Seedlings were treated with glyphosate isopropyl amine at 10-14 leaf stage at rates of 0, 450, 900, 1800 , and $3600 \mathrm{~g}$ ai/ha. The solution of glyphosate isopropyl amine was applied with a compressed air cabinet sprayer at nearly 0.5 bars. The values of growth reduction by $50 \%$ (GR50) were analysed at 16 days after application (daa) with glyphosate, and the shikimic acid contents of $S$. nigrum leaves were determined at 0,1 , and 5 daa with glyphosate, respectively. All these tests were repeated at least three times.

\section{Determination of glyphosate resistance}

Glyphosate resistance level was evaluated by three methods, namely, the biomass (i.e., fresh weight of plant) of each biotype, the comprehensive index of pesticide injury, and the leaf disc.

As for the biomass method, the correlation of biomass and applied glyphosate rate was complied with the three-parameter log-logistic nonlinear dose-response model ${ }^{14-16}$ according to the following formula:

$$
y=\frac{d}{1+\exp (b \log x-\log e)}
$$

where $y$ is the biomass, $x$ the applied glyphosate rate, $d$ the difference between the maximum and the minimum points for the curve, $b$ the slope of the curve, and $e$ the herbicide rate which gave 50\% growth reduction (GR50). Data were calculated with LL.3() function in the main function 'drm' of 'drc' package in R3.4.3. This log-logistic model is convenient and advantageous, because the GR50 values can be estimated directly and the fitting degree of the model can be judged by $p$ value through $t$-Test.

The comprehensive index of pesticide injury was calculated according to the following formula:

$$
i=1-\frac{\Sigma L N}{5 T}
$$

where $i$ is the comprehensive index of pesticide injury, $L$ the level of injury (value is an integer ranging from 1-5), $N$ the plant number observed at the level, and $T$ the total plant numbers in each test. Then the GR50 values were regressed according to formula (1). Briefly, the level of injury of $S$. nigrum was estimated according to the following standards ${ }^{17}$ :

1: The central leaf was normal or was only slightly withering;

2: The central leaf was withering and drooping; the tip of central leaf was rolling; the margin of central leaf was yellowing; and the whole plant was withering;

3: The central leaf was rolling and yellowing, and the area of withered leaves was accounted for $40-70 \%$ of the whole;

4: The central leaf was rolling and yellowing, and the area of withered leaves was accounted for more than $70 \%$ of the whole; 
5: The whole plant was withered, and the area of withered leaves was accounted for more than $95 \%$ of the whole.

The fold of glyphosate resistance was calculated through GR50 for the resistant biotype divided by GR50 for the susceptible biotype.

The leaf disc method was in accordance with the research of Zhang et al ${ }^{18}$ with modification. In brief, the $S$. nigrum leaves at 10-14 leaf stage were punched to the round discs with $1.2 \mathrm{~cm}$ in diameter by a manual puncher. Then the round discs were put into Petri dishes and bred with $900 \mathrm{~g}$ ai/ha of glyphosate for $72 \mathrm{~h}$ at the ambient temperature. Control was cultivated with distilled water at the same condition. This experiment was repeated three times.

\section{Analysis of shikimic acid content}

The shikimic acid content of $S$. nigrum leaves was analysed on the basis of the research of Gaitonde and Gordon ${ }^{19}$ and Lou et al ${ }^{20}$ with modification. Seedlings at 10-14 leaf stage were applied with $450 \mathrm{~g}$ ai/ha glyphosate and bred outdoors. The shikimic acids of leaves were determined at 0,1 , and 5 daa, respectively. In brief, fresh leaves $(0.2 \mathrm{~g})$ were grinded at $4^{\circ} \mathrm{C}$ with $0.6 \mathrm{ml} 0.25 \mathrm{M} \mathrm{HCl}$, and then centrifuged at $12000 \mathrm{~g}$ at $4^{\circ} \mathrm{C}$ for $20 \mathrm{~min}$. After centrifugation, the supernatant was collected and set to the total volume of $1.0 \mathrm{ml}$ with $0.25 \mathrm{M} \mathrm{HCl}$, and then stored at $4^{\circ} \mathrm{C}$ until use. The supernatant (100 $\mu \mathrm{l})$ was added with $1 \mathrm{ml} 1 \%$ periodic acid and stored at ambient temperature for $3 \mathrm{~h}$. Then $1 \mathrm{ml}$ $1 \mathrm{M} \mathrm{NaOH}$ and $0.6 \mathrm{ml} 0.1 \mathrm{M}$ glycine were mixed to the solution and reacted at ambient temperature for $5 \mathrm{~min}$ before the absorbance was detected at $380 \mathrm{~nm}$ with a UV spectrophotometer (Model UV-1240, Shimadzu, Tokyo). The standard curve was determined by using the shikimic acid of high grade instead of the supernatant and analysed as mentioned above.

\section{RESULTS}

\section{Analysis of glyphosate resistance of $S$. nigrum}

S. nigrum of the 's' population was satisfactorily controlled by glyphosate, and the demanding rate was $637 \mathrm{~g}$ ai/ha for $50 \%$ control (GR50) by the biomass method. Whereas the suspected populations from ' $\mathrm{r} 1-\mathrm{r} 10$ ' whose seeds were collected from the agricultural field areas showed resistances to glyphosate which were less than 5 -fold as compared to the ' $\mathrm{s}$ ' population (Table 1). Among these populations, the ' $r 1$ ' population exhibited the highest resistance with GR50 value up to $2751 \mathrm{~g}$ ai/ha, and the resistance
Table 1 Glyphosate resistance levels $(x)$ of $S$. nigrum evaluated by biomass.

\begin{tabular}{lccc}
\hline Pop. & GR50 (g ai/ha) & Regression equation & $x$ \\
\hline $\mathrm{s}$ & $637 \pm 42$ & $y=\frac{53.48}{1+\exp (0.9707 \log x-\log 637)}$ & $1.00^{*}$ \\
$\mathrm{r} 1$ & $2751 \pm 174$ & $y=\frac{53.03}{1+\exp (1.4551 \log x-\log 2751)}$ & $4.32^{*}$ \\
$\mathrm{r} 2$ & $1650 \pm 101$ & $y=\frac{50.60}{1+\exp (1.6436 \log x-\log 1650)}$ & $2.59^{*}$ \\
$\mathrm{r} 3$ & $1603 \pm 130$ & $y=\frac{51.29}{1+\exp (0.9426 \log x-\log 1603)}$ & $2.52^{*}$ \\
r4 & $1247 \pm 42$ & $y=\frac{55.55}{1+\exp (1.4228 \log x-\log 1247)}$ & $1.96^{*}$ \\
r5 & $1018 \pm 92$ & $y=\frac{52.92}{1+\exp (1.1272 \log x-\log 1018)}$ & $1.60^{*}$ \\
r6 & $1168 \pm 66$ & $y=\frac{53.45}{1+\exp (1.5689 \log x-\log 1168)}$ & $1.83^{*}$ \\
r7 & $1045 \pm 45$ & $y=\frac{56.48}{1+\exp (1.3087 \log x-\log 1045)}$ & $1.64^{*}$ \\
r8 & $1032 \pm 34$ & $y=\frac{51.39}{1+\exp (1.5712 \log x-\log 1032)}$ & $1.62^{*}$ \\
r9 & $1990 \pm 78$ & $y=\frac{52.38}{1+\exp (1.3479 \log x-\log 1990)}$ & $3.12^{*}$ \\
r10 & $1299 \pm 74$ & $y=\frac{53.29}{1+\exp (1.2357 \log x-\log 1299)}$ & $2.04^{* *}$ \\
\hline
\end{tabular}

' $\mathrm{s}$ ' is the susceptible population, and ' $\mathrm{r} 1-\mathrm{r} 10$ ' are the suspected glyphosate resistant populations. Regression was calculated according to the three-parameter log-logistic nonlinear dose-response model.

$*, p<0.0001 ; * *, p<0.001$.

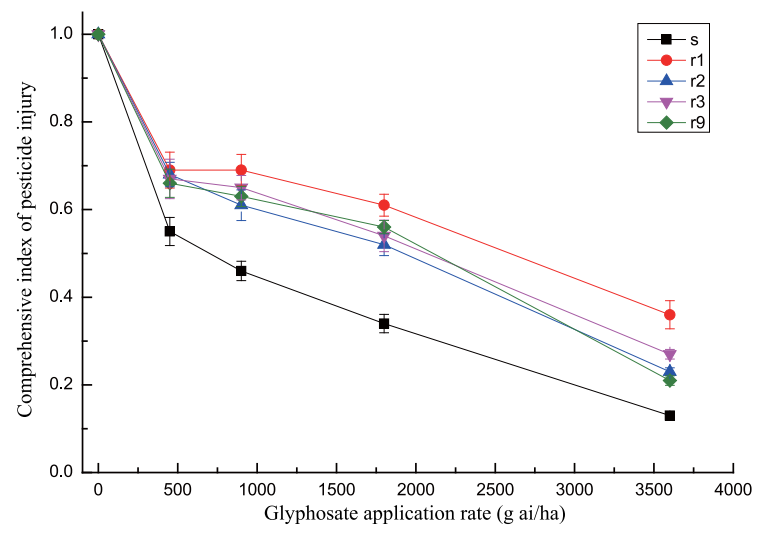

Fig. 1 Log-logistic dose-response curve of the 's', ' $r 1, r 2$, r3, and r9' populations of $S$. nigrum from Hunan Province of China by the comprehensive index of pesticide injury analysis. All the tests were performed in triplicate and expressed as mean \pm standard error.

of the ' $r 9$ ' population was over 3-fold compared with that of the 's' population. The resistance of the ' $r 2$, r3, and r10' populations was over 2-fold compared with that of the 's' population, whereas the others showed less than 2 -fold compared with that of the 's' population.

As for the determination of glyphosate resistance through the comprehensive index of pesti- 
Table 2 Glyphosate resistance levels $(x)$ of $S$. nigrum evaluated by comprehensive index of pesticide injury.

\begin{tabular}{lccc}
\hline Pop. & GR50 (g ai/ha) & Regression equation & $x$ \\
\hline $\mathrm{s}$ & $660 \pm 51$ & $y=\frac{0.9982}{1+\exp (0.8681 \log x-\log 660)}$ & $1.00^{*}$ \\
$\mathrm{r} 1$ & $1798 \pm 247$ & $y=\frac{0.9930}{1+\exp (0.8304 \log x-\log 1798)}$ & $2.72^{*}$ \\
$\mathrm{r} 2$ & $1444 \pm 130$ & $y=\frac{0.9941}{1+\exp (0.9318 \log x-\log 1444)}$ & $2.19^{*}$ \\
$\mathrm{r} 3$ & $1580 \pm 166$ & $y=\frac{0.9946}{1+\exp (0.8183 \log x-\log 1580)}$ & $2.39^{*}$ \\
$\mathrm{r} 4$ & $973 \pm 48$ & $y=\frac{0.9987}{1+\exp (0.7298 \log x-\log 973)}$ & $1.47^{*}$ \\
$\mathrm{r} 5$ & $782 \pm 22$ & $y=\frac{0.9994}{1+\exp (0.8663 \log x-\log 782)}$ & $1.19^{*}$ \\
$\mathrm{r} 6$ & $796 \pm 90$ & $y=\frac{0.9968}{1+\exp (0.8683 \log x-\log 796)}$ & $1.21^{*}$ \\
$\mathrm{r} 7$ & $796 \pm 74$ & $y=\frac{0.9967}{1+\exp (0.9009 \log x-\log 796)}$ & $1.21^{*}$ \\
$\mathrm{r} 8$ & $874 \pm 86$ & $y=\frac{0.9962}{1+\exp (0.9445 \log x-\log 874)}$ & $1.32^{*}$ \\
$\mathrm{r} 9$ & $1451 \pm 198$ & $y=\frac{0.9925}{1+\exp (0.8841 \log x-\log 1451)}$ & $2.20^{*}$ \\
$\mathrm{r} 10$ & $861 \pm 81$ & $y=\frac{0.9972}{1+\exp (0.8491 \log x-\log 861)}$ & $1.30^{*}$ \\
\hline & &
\end{tabular}

${ }^{*}, p<0.0001$.

cide injury method, we found that the index of pesticide injury was also well in accordance with the rate of pesticide with the log-logistic model. When GR50 was calculated with regression, the $p$ values were always less than 0.0001 , exhibiting a satisfactory fitting degree to the log-logistic model. The results with this method were similar to those of the biomass method, but the resistance fold values against glyphosate were a little lower than those of the biomass method, and only the ' $r 1, r 2, r 3$, and r9' populations showed more than 2-fold of glyphosate resistance compared with the 's' population (Fig. 1, Table 2).

The glyphosate resistance was also evaluated through the leaf disc method in this study. The results of discs of $S$. nigrum leaves applied with glyphosate were also in accordance with those of the above two methods. The discs of ' $r 1, r 2, r 3$, and r9' populations remained green after application with glyphosate, whereas others began withering and turned yellow, indicating that these populations had higher resistances against glyphosate than the others (Fig. 2).

\section{Shikimic acid content of $S$. nigrum}

As shown in Table 3, the background levels of shikimic acid were from 8.75-20.17 $\mu \mathrm{g} / \mathrm{g}$ fresh weight before treated with glyphosate, depending on the different sources of $S$. nigrum seeds. The level of shikimic acid of the 's' population increased 1.28fold at 5 daa treatment compared to the untreated tissue. In the suspected glyphosate resistant pop-

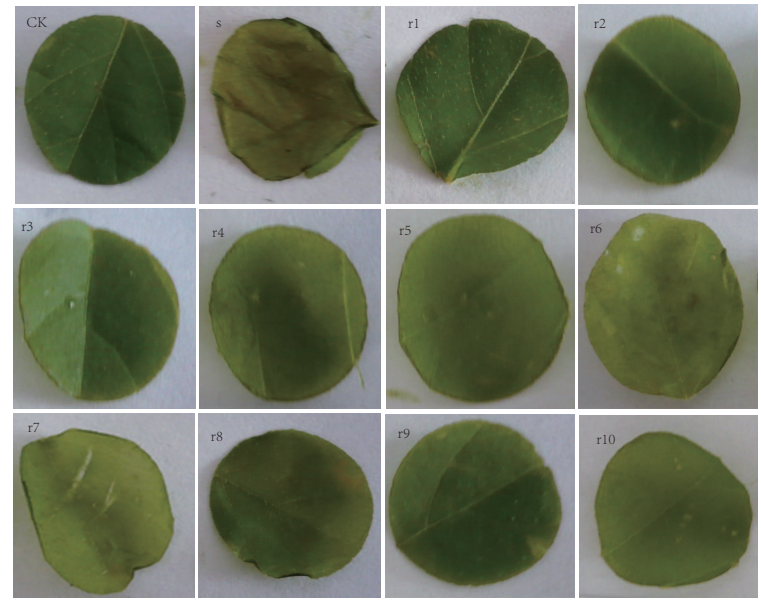

Fig. 2 Discs of $S$. nigrum leaves applied with glyphosate. CK was applied with distilled water, and the 's' and 'r1-r10' populations were applied with $900 \mathrm{~g}$ ai/ha of glyphosate for $72 \mathrm{~h}$ at ambient temperature.

Table 3 Shikimic acid content of $S$. nigrum leaves after applied with glyphosate.

\begin{tabular}{lccc}
\hline \multirow{4}{*}{ Pop. } & \multicolumn{3}{c}{ Shikimic acid content $(\mu \mathrm{g} / \mathrm{g}$ fresh weight $)$} \\
\cline { 2 - 4 } & 0 daa & 1 daa & 5 daa \\
\hline s & $11.95 \pm 1.50^{\mathrm{b}}$ & $13.07 \pm 0.53^{\mathrm{b}}$ & $39.15 \pm 2.78^{\mathrm{a}}$ \\
r1 & $19.98 \pm 0.52^{\mathrm{a}}$ & $17.43 \pm 0.95^{\mathrm{b}}$ & $13.78 \pm 1.21^{\mathrm{c}}$ \\
r2 & $20.17 \pm 2.46^{\mathrm{a}}$ & $18.60 \pm 1.68^{\mathrm{ab}}$ & $13.03 \pm 3.98^{\mathrm{b}}$ \\
r3 & $17.27 \pm 0.88^{\mathrm{a}}$ & $18.78 \pm 2.02^{\mathrm{a}}$ & $17.87 \pm 0.73^{\mathrm{a}}$ \\
r4 & $19.38 \pm 1.01^{\mathrm{a}}$ & $12.15 \pm 0.19^{\mathrm{b}}$ & $18.13 \pm 0.52^{\mathrm{a}}$ \\
r5 & $11.35 \pm 0.39^{\mathrm{b}}$ & $15.43 \pm 0.42^{\mathrm{b}}$ & $22.80 \pm 4.85^{\mathrm{a}}$ \\
r6 & $12.78 \pm 0.59^{\mathrm{b}}$ & $19.73 \pm 0.51^{\mathrm{b}}$ & $60.92 \pm 5.79^{\mathrm{a}}$ \\
r7 & $8.75 \pm 0.19^{\mathrm{b}}$ & $13.25 \pm 0.21^{\mathrm{b}}$ & $18.48 \pm 0.27^{\mathrm{a}}$ \\
r8 & $9.75 \pm 1.20^{\mathrm{c}}$ & $14.08 \pm 0.25^{\mathrm{b}}$ & $24.40 \pm 0.68^{\mathrm{a}}$ \\
r9 & $11.37 \pm 0.43^{\mathrm{b}}$ & $14.67 \pm 2.02^{\mathrm{b}}$ & $24.33 \pm 1.78^{\mathrm{a}}$ \\
r10 & $14.95 \pm 0.73^{\mathrm{b}}$ & $17.70 \pm 0.83^{\mathrm{b}}$ & $31.17 \pm 3.13^{\mathrm{a}}$ \\
\hline
\end{tabular}

All the tests were performed in triplicate and expressed as mean \pm standard error. Significant analysis was carried with SPSS19.0 with ANOVA (Duncan test) and marked with lowercase letters.

ulations, the levels of shikimic acid showed three different trends. The levels of shikimic acid of the ' $r 5-r 10$ ' populations increased, whereas shikimic acid of the ' $r 3$ and $r 4$ ' populations kept the same levels at 5 daa treatment, and those of the ' $r 1$ and r2' populations decreased compared with the untreated tissues at 5 daa treatment. In this study, we observed that the levels of shikimic acid among the suspected glyphosate resistant populations did not respond to an expected normal sigmoidal curve 
to their glyphosate resistance folds.

\section{DISCUSSION}

The identification of the $S$. nigrum biotypes with resistance or tolerance to glyphosate in Hunan Province of China has not been previously documented. In recently years, we have observed the phenomenon of resistance to glyphosate among the $S$. nigrum biotypes. For example, in the agricultural fields such as $C$. oleifera and yellow peach orchards during their juvenile stage of trees, the broadleaf weed $S$. nigrum was found not well controlled under the commercial rates of glyphosate.

Weed resistance to herbicide is often evaluated by GR50 or LD50 (lethal dose to $50 \%$ of the population) values calculated by the log-logistic model of dose-response curves ${ }^{11,16}$. Thus to obtain GR50 values, two independent experiments including the biomass method and the comprehensive index method were designed to analyse the glyphosate resistance of $S$. nigrum seeds from different agricultural fields of Hunan Province.

The three-parameter log-logistic model provides the appropriate analysis of data where the lower limit is equal to 0 , with the significant model fit by $t$-Test ${ }^{21}$. In this study, the $p$ values were always less than 0.0001 , indicating a satisfactory fitting degree of the curves was achieved in each test (Tables 1 and 2). The GR50 value of the 's' population through the biomass method was in perfect agreement compared with the values from the comprehensive index method, which were $637 \mathrm{~g}$ ai/ha and $660 \mathrm{~g}$ ai/ha, respectively. In addition, the GR50 values of the suspected resistant populations through the biomass method exhibited the similar trends compared with those of the comprehensive index method. These results were consistent with the former studies in Nanjing Agriculture University on horseweed resistance to glyphosate ${ }^{22}$. It should be mentioned that the GR50 values of the suspected glyphosate resistant populations of $S$. nigrum from the comprehensive method were lower compared with those of the biomass method, possibly because the subjective errors during observations might be produced in judging the damage levels caused by glyphosate. In addition, by comparison of the suspected glyphosate resistant populations of $S$. nigrum with the susceptible biotype, the results from the biomass method, the comprehensive index method, and the leaf disc method could lead to a preliminary conclusion that the ' $r 1, r 2, r 3$, and r9' populations were the moderate glyphosate resistant biotypes from Hunan Province of China according to the standard put forward by the Weed Science Society of America ${ }^{3}$, because they were not well controlled by a relatively high rate of commercial glyphosate application, and then they performed less than 10fold resistance relative to the 's' population.

Since shikimic acid is the substrate of EPSP synthase as for a precursor for the biosynthesis of aromatic acids in plants ${ }^{23}$, its content can reflect the activity condition of EPSP synthase. Glyphosate can induce plants to accumulate shikimic acid. Thus the measurement of shikimic acid accumulation in response to glyphosate is often considered as a quick assay to evaluate the plant resistance ${ }^{24,25}$. Early research showed that the shikimic acid levels in horseweed displayed a typical sigmoidal curve to glyphosate resistance ${ }^{26}$. In this study, based on the determination of the shikimic acid assay at 5 daa treatment, the shikimic acid contents of the ' $r 1$, r2, and $r 3$ ' populations were the same as or lower than those of untreated tissues, whereas the shikimic acid content of the 'r9' population was over 2-fold compared with that of untreated tissue. However, based on GR50 assay, the glyphosate resistance of the ' $r 1$ ' population was greater than the others, and the glyphosate resistances of ' $r 2$, $r 3$, and r9' populations were nearly at a similar level. The relation of shikimic acid level to glyphosate resistance did not show a typical sigmoidal curve, implying that different resistance mechanism might be involved among the glyphosate resistant populations of $S$. nigrum.

\section{CONCLUSION}

In this study, four glyphosate resistance populations were identified including the ' $r 1, r 2, r 3$, and r9' populations of $S$. nigrum from ten suspected glyphosate resistant populations collected in $\mathrm{Hu}-$ nan Province of China which had not been documented before. In addition, they were confirmed as moderate glyphosate resistant biotypes by the biomass method, the comprehensive index method, and the leaf disc method. But the levels of shikimic acid to glyphosate resistances among the suspected glyphosate resistant populations did not respond to a typical sigmoidal curve, indicating that different resistance mechanism might be involved among the glyphosate resistant biotypes.

Acknowledgements: This work was supported by the Scientific Research Fund of Hunan Provincial Education Department (15K066, 16K047) and the Research and Innovation Project of Postgraduate Students of Hunan Province (CX2016B677). 


\section{REFERENCES}

1. Yu Q, Jalaludin A, Han H, Chen M, Sammons RD, Powles S (2015) Evolution of a double amino acid substitution in the 5-enolpyruvylshikimate-3phosphate synthase in Eleusine indica conferring high-level glyphosate resistance. Plant Phsyiol 167, 1440-1447.

2. Heap I (2018) The international survey of herbicide resistant weeds. Weed Science Society of America.

3. Gaines TA, Heap IM (2018) Mutations in herbicideresistant weeds to EPSP synthase. Weed Science Society of America.

4. Johnson WG, Davis VM, Kruger GR, Weller SC (2009) Influence of glyphosate-resistant cropping systems on weed species shifts and glyphosate-resistant weed populations. Eur J Agron 31, 162-172.

5. Busi R, Powles SB (2009) Evolution of glyphosate resistance in a Lolium rigidum population by glyphosate selection at sublethal doses. Heredity 103, 318-325.

6. Wu Q, Leung JYS, Huang X, Yao B, Yuan X, Ma J, Guo S (2015) Evaluation of the ability of black nightshade Solanum nigrum L. for phytoremediation of thallium-contaminated soil. Environ Sci Pollut Res 22, 11478-11487.

7. Istiaji RP, Fitria M, Larasati, Tjondro F, Maruti AA, Setyowati EP, Meiyanto E (2010) Leunca (Solanum nigrum L.) herbs ethanolic extract increase cytotoxic activity of cisplatin on Hela cervical cancer cells. Indonesian J Cancer Chemoprevention 1, 32-37.

8. Jani DK, Ahir KB (2010) Kakamachi (Solanum nigrum Linn.) - A prominent herb in Ayurveda. Life Sciences Leaflets 9, 234-240.

9. Holm LG, Plucknett DL, Pancho JV, Herberger JP (1977) Solanum nigrum L. In: The World's Worst Weeds: Distribution and Biology, University of Hawaii Press, Honolulu, pp 430-434.

10. Huang Y, Zhu Q, Yang X, Ji H (2016) Determination of total alkaloids in Solanum nigrum by acid dye colorimetry. Med Plant 7, 42-44.

11. de Carvalho SJP, Netto AG, Nicolai M, Cavenaghi AL, López-ovejero RF, Christoffoleti PJ (2015) Detection of glyphosate-resistant palmer amaranth (Amaranthus palmeri) in agricultural areas of Mato Grosso, Brazil. Planta Daninha 33, 579-586.

12. López-ovejero RF, Soares DJ, Oliveira WS, Fonseca LB, Berger GU, Soteres JK, Christoffoleti PJ (2013) Residual herbicides in weed management for glyphosate-resistant soybean in Brazil. Planta Daninha 31, 947-959.
13. Page E, Granger C, Tardif F, Rajcan I, Laforest M, Nurse RE (2017) A chrono-geographical assessment of Canadian populations of Conyza canadensis. WSSA Proceedings 57, 259.

14. Ritz C, Baty F, Streibig JC, Gerhard D (2015) Doseresponse analysis using R. PloS One 10, e0146021.

15. van Gess MJ (2001) Glyphosate-resistant horseweed from Delaware. Weed Sci 49, 703-705.

16. Vargas L, Ulguim AR, Agostinetto D, Magro TD, Thürmer L (2013) Low level resistance of goosegrass (Eleusine indica) to glyphosate in Rio Grande do SulBrazil. Planta Daninha 31, 677-686.

17. Wu J (2006) Comparative evaluation of efficacy of different formulations of glyphosate and monitoring of glyphosate-resistant (or tolerant) weeds. Nanjing Agricultural University, Nanjing, China.

18. Zhang Z, Zhou Z, Liu Y, You M (1994) Obtainment of CMVcp transgene-tobacco-plants by means of leaf disc methods. $J$ Tianjin Inst Technol 10, 79-82.

19. Gaitonde MK, Gordon MW (1958) A microchemical method for the detection and determination of shikimic acid. J Biol Chem 230, 1043-1050.

20. Lou Y, Deng Y, Shen J, Xu L, Shen W (2005) Effects of metsulfuron-methyl and glyphosate on acetolactate synthase activities and shikimate levels of Alternanthera philoxeroides. Acta Phytophy Sin 32, 185-188.

21. der Vliet LV, Ritz C (2013) Statistics for analyzing ecotoxicity test data. In: Férard J, Blaise C (eds) Encyclopedia of Aquatic Ecotoxicology, Springer, New York, pp 1081-1096.

22. Wu J, Sun X, Qiang S, Gen H (2006) Establishment of detection method of glyphosate resistant horseweed. Jiangsu Agr Sci 6, 187-189.

23. Borah JC (2015) Shikimic acid: a highly prospective molecule in pharmaceutical industry. Curr Sci 109, 1672-1679.

24. Becerra-Moreno A, Benevides J, Cisneros-Zevallos L, Jacobo-Velázquez DA (2012) Plants as biofactories: glyphosate-induced production of shikimic acid and phenolic antioxidants in wounded carrot tissue. $J$ Agric Food Chem 60, 11378-11386.

25. Pline WA, Wilcut JW, Duke SO, Edmisten KL, Wells R (2002) Tolerance and accumulation of shikimic acid in response to glyphosate applications in glyphosate-resistant and nonglyphosate-resistant cotton (Gossypium hirsutum L.). J Agric Food Chem 50, 506-512.

26. Main CL (2005) Investigation of glyphosate-resistant horseweed (Conyza canadensisw L. Cronq.) in Tennessee. University of Tennessee, Knoxville, USA. 